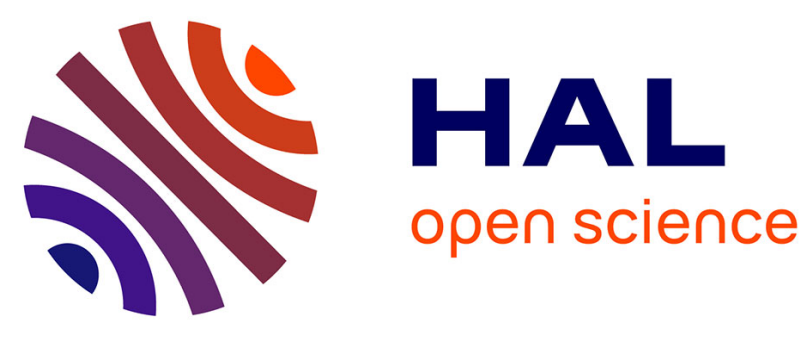

\title{
Lettres fictives à son juge
}

Mathias Gardet

\section{To cite this version:}

Mathias Gardet. Lettres fictives à son juge: Les dossiers de jeunes délinquants du centre public d'observation de Savigny-sur-Orge, 1950-1965. Les cahiers dynamiques, 2010, 46, p. 111 à 118. hal02966486

\section{HAL Id: hal-02966486 \\ https://hal-univ-paris8.archives-ouvertes.fr/hal-02966486}

Submitted on 14 Oct 2020

HAL is a multi-disciplinary open access archive for the deposit and dissemination of scientific research documents, whether they are published or not. The documents may come from teaching and research institutions in France or abroad, or from public or private research centers.
L'archive ouverte pluridisciplinaire HAL, est destinée au dépôt et à la diffusion de documents scientifiques de niveau recherche, publiés ou non, émanant des établissements d'enseignement et de recherche français ou étrangers, des laboratoires publics ou privés. 


\title{
Lettres fictives à son juge, \\ Les dossiers de jeunes délinquants du centre public d'observation de Savigny-sur-Orge, 1950-1965
}

\author{
par Mathias Gardet, historien, PR en sciences de l'éducation, université de Paris 8 \\ publié dans Les cabiers dynamiques, nº46, avril 2010, p. 11-118
}

\begin{abstract}
Monsieur le juge, dépôt de Paris
Monsieur,

Je vous écris une lettre pour vous parler du placement que vous m'avez. offert. Je trouve que ce placement est bien, mais la punition que vous m'avez faite est peut-être un peu dure. Surtout que c'est simplement une fugue de chez. mes parents, qui n'a duré que quatre jours. Mes parents étant décidés à me reprendre, j’espère que vous me ferez libérer le plus vite possible.

En espérant une réponse favorable recevez. Monsieur mes salutations et respects Signature ${ }^{1}$
\end{abstract}

Contrairement aux apparences, cette citation, choisie parmi tant d'autres dans les dossiers de mineurs confiés par les tribunaux au centre d'observation de Savigny-sur-Orge, n'est pas une vraie lettre. Le juge des enfants, parfois interpellé nommément par les mineurs auteur de ces écrits, n'en prendra jamais connaissance. Non pas parce que la correspondance aurait été mal acheminée ou interceptée, mais tout simplement parce que c'est un exercice fictif consigné sur un cahier d'écolier. Le jeune qui rédige sait très bien lui-même qu'il s'agit d'une fausse missive, que la feuille ne sera jamais arrachée du cahier pour être glissée dans une enveloppe, même s'il prend parfois la peine de dessiner la dite enveloppe dans la marge avec son faux timbre. Il sait que c'est un artifice, puisqu'il se prête à un devoir particulier en répondant à un sujet imposé de rédaction standard donné à tous ses camarades placés dans le centre sur le thème : «Tu écris au juge pour lui dire ce que tu penses de ton placement à Savigny». S'imagine-t-il en revanche que le juge feuillettera son cahier et que donc son message lui parviendra, c'est peu probable, et je ne pense pas que ce facteur soit décisif dans la motivation de l'auteur, ni dans le ton employé, ni dans le choix des mots. Ces rédactions ne sont d'ailleurs pas organisées sous forme de plaidoirie, de justification dans un désir de dédouanement ou de réparation, mais plus sous forme de composition de français laissant libre cours à l'imagination. Qui dit appel à l'imaginaire ne veut cependant pas dire récit fantaisiste. Si une partie des sujets de rédactions proposés font ouvertement appel à la fabulation ou à la métaphore, la plupart sont ancrés plus ou moins directement dans une réalité quotidienne et les jeunes puisent souvent dans leur vécu pour y répondre sans avoir l'impression de se soumettre à un interrogatoire. Malgré l'ombre tutélaire du maitre-correcteur, dans sa copie, l'élève ne s'adresse pas une personne précise ou alors elle est éloignée, mise à distance. Durant toute sa scolarité dans le cycle primaire, il a été habitué, presque conditionné, à s'adresser à des interlocuteurs inventés pour le besoin de l'exercice: "la coopérative scolaire organise une tombola. Vous écrivez à un commerçant ami de l'école pour lui demander un lot », "lettre à votre mère pour la prévenir que vous êtes en retenue ", "écrivez à votre cousine qui habite près de Paris, pour l'inviter, ainsi que ses parents, à venir passer chez vous, la journée du mardi-gras »...2.

\footnotetext{
1. J'ai fait le choix de ne pas reproduire les fautes d'orthographes qui n'ont de sens que dans leur aspect visuel, tout en laissant les tournures de phrases.

2. Exemples de rédactions cités dans Brigitte Dancel, Un siècle de rédactions : Ecrits d'écoliers et de collégiens, CRDP de l'académie de Grenoble, 2001, p. 58, 60, 131.
} 
Astreint à l'écriture durant des après-midi entiers, le mineur placé à Savigny-sur-Orge, retrouve donc ses réflexes d'écolier. Le silence étant de rigueur, il est seul avec lui-même devant la feuille blanche et finit paradoxalement par s'emparer de ce temps infligé pour libérer sa parole, comme le montre par exemple ces extraits tirés d'un même cahier en décembre 1952 :

Écris sur ton cahier toutes les réflexions que depuis une semaine la vie au centre a pu te suggérer : En classe l'éducateur distribue des fenillets où l'on inscrit son nom, son prénom et sa date de naissance, et l'on attend l'ordre de tourner les pages, lorsqu'un devoir est terminé, il faut porter son cabier au pupitre, pour en recevoir un deuxième. Il y a du français et du calcul. Tout ce qu'il faut pour entretenir la mémoire des principales règles arithmétiques, de dessin et surtout des rédactions. On commence à reprendre l'babitude de manier le porte-plume, le crayon, la gomme et à reprendre de l'encre sur le papier sans raison, rien que pour entretenir l'habitude d'écrire...

Ecris sur deux feuilles de ton cahier tout ce qui te passe par la tête, sans réfléchir :

J'ai une envie folle de fiche en l'air, cabier, crayon, gomme, encrier, surveillant et tout le monde, je voudrais voir le centre et tout ce qu'ily a dedans au cinq cents diables. Je voudrais être chę moi, les pieds dans les pantoufles, au coin du feu, en fumant quelques bonnes Balto...

Malgré la forte référence au monde de l'école, renforcé par le support choisi pour ces écrits - des petits cahiers, format $17 \mathrm{~cm} \mathrm{X} \mathrm{22cm,} \mathrm{de} \mathrm{la} \mathrm{marque} \mathrm{«Clair} \mathrm{matin} \mathrm{»} \mathrm{ou} \mathrm{bien} \mathrm{«Hermès} \mathrm{»} \mathrm{ou} \mathrm{bien}$ encore "Chambord», avec leurs pages à grands carreaux, une ligne rouge sur la gauche pour démarquer la marge -, ces textes ne sont pas des exercices scolaires. Détournés de leur vocation d'origine, ces sujets de rédaction deviennent partie prenante de tout l'arsenal de tests psychologiques auquel sont soumis les jeunes placés dans des centres comme Savigny-sur-Orge, durant ce bref temps de l'observation. Cette observation est orchestrée par le médecin psychiatre et ses adjoints psychologues ${ }^{3}$, qui mobilisent l'ensemble des intervenants prenant en charge le mineur durant son séjour pour rédiger au bout de trois mois un rapport d'une quinzaine de pages, résumé en une synthèse de deux pages. Si dans ce document les propos du jeune sont cités, il ne s'agit que de morceaux de phrases épars, jugés symptomatiques de ses troubles du comportement, et accompagnés d'une grille de lecture basée sur une étiologie psychologisante ${ }^{4}$.

Le juge des enfants ne fait pas partie de ce processus, il a un bref entretien en amont avec le jeune, qui a motivé pour beaucoup d'entre eux un envoi à Savigny-sur-Orge, en vertu d'une ordonnance de placement provisoire (OPP) ; il le revoit en aval, trois mois plus tard. Lors de ce second entretien, il décide la plupart du temps soit une remise à la famille sur simple admonestation, soit de le confier à la garde « d'une oeuvre privée ou placé dans un établissement ou dans une institution d'éducation, de formation professionnelle ou de soins, dans un institut médico-pédagogique, de l'État ou d'une administration publique, dans une institution d'éducation professionnelle, d'éducation surveillée ou d'éducation corrective», pour une durée qui "ne pourra excéder l'époque où il aura atteint l'âge de vingt et un ans $\aleph^{5}$. Or, pour prononcer ce jugement, il n'a eu entre les mains que la synthèse succincte issue de l'observation. Le volumineux dossier où ont été engrangés les divers documents qui ont permis d'élaborer ce pronostic a été conservé par le centre de Savigny après le départ du jeune, et finit par être empilé avec ses prédécesseurs, par liasses trimestrielles, dans un des greniers de l'institution. Sauf très rares exceptions, il ne sera plus jamais consulté, ainsi que l'atteste la couche de poussière déposée sur les piles de dossiers jaunis, jusqu'à leur toute récente exhumation pour un versement aux archives départementales de l'Essonne.

\footnotetext{
3. La psychologie ne s'est imposée comme discipline universitaire à part entière que depuis 1947 et les premiers psychologues travaillant à l'Education surveillée sont encore souvent des passeurs de tests. Annick Ohayon, L'impossible rencontre. Psychologie et psychanalyse en France (1919-1969), Paris, La Découverte, 1999, 438 p.

4. Jean-Claude Chamborédon, "La délinquance juvénile, essai de construction d'objet », Revue française de sociologie, XII-3, 1971, p. 335.

5. Article 66 du codé pénal modifié par l'ordonnance du 2 février 1945.
} 
En dehors du contact direct qu'il a eu à deux reprises avec le jeune, le juge des enfants n'a donc eu aucune connaissance de ses réactions, opinions, commentaires retranscrits d'une écriture plus ou moins brouillonne, dans un ou deux petits cahiers d'écolier... Quel dommage !

Ces écrits provoqués, mais paradoxalement spontanés, offre en effet un miroir d'une étonnante acuité sur le ressenti des jeunes face à l'appareil judiciaire. Ils permettent de s'interroger sur le décalage existant - sans doute inévitable - entre l'intention du législateur, la marge de manœuvre du magistrat et la perception du mineur qui s'y trouve confronté, comme le montre par exemple cette rédaction du 31 janvier 1961 :
Mr le juge,
Je ne vois pas pourquoi que vous m'avez. envoyé à Savigny je pense que cela est inutile. Je préférerais attendre mon jugement au dépôt et qu'ensuite on m'envoie à Fresnes ${ }^{6}$ pour faire une peine déterminée tandis que qu'à Savigny nous sommes jamais fixés sur le temps de notre séjour tandis qu'en prison nous savons quand nous rentrons et quand nous sortons.
Naturellement le séjour à Savigny doit être moins dur qu'à Fresnes et la nourriture pas la même, mais, J'estime que la liberté compte plus que n'importe quoi
$M r$ le juge je termine ma lettre en espérant que vous m'avez compris et en vous envoyant mes salutations distinguées

Cette incompréhension exprimée face à un jugement qui, dans l'esprit de l'ordonnance de 1945, recherche une meilleure adéquation de la mesure éducative plutôt qu'une punition par enfermement, renvoie en abîme à l'effet pervers des article du code pénal en matière de Justice des mineurs, déjà dénoncé par des observateurs depuis la fin du XIX siècle :

«Les condamnations prononcées contre les mineurs de 16 ans $^{7}$ ne portent en général que des peines de courtes durées, en raison des réductions de pénalités dont le code pénal leur assure le bénéfice. Ces condamnations sont, en moyenne, d'un an et demi à deux ans d'emprisonnement. Les envois en correction sont, au contraire, généralement prononcés par les tribunaux jusqu'à seize, dix-huit et même vingt ans ; c'est-à-dire, en général, pour un temps qui excède la durée des condamnations les plus longues. Il en résulte que les jeunes acquittés ${ }^{8}$, voyant arriver à la colonie de condamnés qui, entrés à la maison après eux, sont généralement libérés avant eux, sont naturellement amenés à porter envie à leur situation. Peu à peu l'idée germe dans leurs jeunes têtes qu'il vaut mieux être condamné qu'acquitté, et cette idée peut faire d'assez grands ravages dans des intelligences et des consciences confuses, où le sentiment du bien et du mal n'est qu'imparfaitement développé... ${ }^{9}{ }^{\prime}$

La portée éducative de certaines mesures, l'évaluation différenciée des situations individuelles des jeunes déférés devant les tribunaux, qui bouleverse l'équation entre les type de délits et le barème des peines, les pratiques personnalisées de tel ou tel juge des enfants ou bien d'un même juge d'un cas sur l'autre, qui n'offre pas toujours une grande cohérence d'application, d'autant que certains tribunaux souffrent d'engorgement, se heurtent aux « codes judiciaires » incorporés par les prévenus. Car contrairement aux affirmations du vicomte d'Haussonville, malgré leur jeune âge, la plupart des mineurs qui se retrouvent à Savigny-sur-Orge ont leur propre conscience des

\footnotetext{
${ }^{6}$. Pour les mineurs de cette époque Fresnes représentait l'archétype de la prison, alors que paradoxalement, vu son âge, il aurait pu se retrouver au Centre spécial d'observation de l'éducation surveillée (CSOES) de Fresnes, ouvert en 1958, et qui pas plus que Savigny n'aurait proposé d'emblée une peine déterminée.

7. Avant d'être augmentée en 1906 à 18 ans, la majorité pénal était fixée à 16 ans ; la majorité civile étant quant à elle maintenue à 21 ans jusqu'en 1974 qui l'abaisse à 18 ans.

8. La première version de l'article 66 du code pénal de 1810 énonçait que les accusés de moins de 16 ans, s’il était décidé qu'ils avaient agi sans discernement, seraient acquittés, tout en proposant dans la même mesure qu'ils soient placés. La réforme du 2 février 1945 supprime la notion de discernement et d'acquittement, et en laissant le libre arbitre au magistrat de prononcer ou non une condamnation pénale, selon la personnalité du délinquant, elle estompe dans la pratique les distinctions entre l'article 66 et l'article 67.

9 Vicomte Joseph-Othenin-Bernard de Cléron d'Haussonville, Les établissements pénitentiaires en France et aux colonies, Paris, Michel Levy frères-Librairie nouvelle, 1875, pp.320-321
} 
rouages judiciaires et ils ont bien intégré la notion de transgression inhérente au délit commis, ainsi que son corollaire sous forme de peine «à tirer». Cette conscience est souvent construite à partir d'échanges d'expériences dans les salles d'attente ou dans les centres de placement, ou bien est basée sur les récits de ceux qui, avant eux, sont passés par là. Ils n’hésitent pas alors à pointer les disfonctionnements ou ce qui leur apparaît comme de l'arbitraire comme le montre ces extraits de deux cahiers rédigés au début des années 1960 :

Quelle est l'injustice qui t'a fait le plus souffrir ?

L'injustice qui m'a fait le plus souffrir, ce n'est pas d'être atterri à Savigny car je l'ai mérité depuis longtemps, mais c'est de s'être tapé 4 jours au dépôt, d'être passé au juge, m'avoir relâché, et une semaine après venir m'arrêter pour être expédié à Savigny. Je trouve que soit injuste, car lorsque je suis passé au juge il aurait dû m'arrêter tout de suite et non me relâcher et m'arrêter une semaine après...

C'est à la maison de Pontoise où 3 garçons sont rentrés en même temps que mon compagnon de cellule. Les 3 garcons avaient volé 57 voitures et le copain a volé une mobylette. Les 3 gargons sont rentrés chez eux après 4 jours de prison. Et quand mon compagnon de cellule est passé au jugement il a été condamné à 6 mois de prison ferme. Je ne peux pas comprendre cette indulgence pour les uns et cette fermeté pour les autres...

Les délais avant jugement sont vécus comme autant d'incertitudes sur le sort final qui les attend, montrant les incompatibilités entre le temps de l'intervention judiciaire qui doit tenir compte des plannings des salles d'audience et le temps en suspens du prévenu dans l'expectative d'un « verdict». Le sas représenté par Savigny est ainsi plus ressenti comme un purgatoire que comme un échappatoire à la prison car, malgré l'esprit de l'ordonnance du 2 février et le lyrisme de son exposé des motifs, l'héritage pénitentiaire est encore très prégnant. Même si le centre d'observation de Savigny est installé dans les locaux d'une ancienne ferme carrée, les aménagements réalisés par la toute jeune direction de l'Éducation surveillée renvoient inévitablement des images d'enfermement, d'autant que le jeune a souvent passé au préalable, minimum une nuit au dépôt, qu'il a été acheminé au centre en fourgon cellulaire, le fameux panier à salade, et que le comité d'accueil est pour le moins évocateur : vidage des poches, abandon des effets civils, " paquetage comme à l'armée », comme l'évoque par exemple cette rédaction de 1960 sur le thème «En arrivant à Savigny qu'est-ce qui t'ennuie ? » :
"Quand je suis arrivé à Savigny, je me suis trouvé désemparé. On nous enferme comme des criminels. Quand je suis rentré dans ma chambre, j'ai ouvert la petite fenêtre et à moi ce qui me va pas c'est les barreaux qu'ily a. Quand nous sommes dans la salle de jeux, nous sommes pas libres, ily a toujours des barreaux aux fenêtres, on ne peut pas sortir dehors, la porte est fermée à clé... 》

Les écrits de jeunes de Savigny permettent par ailleurs de souligner les différences existantes dans l'appréhension spatiale des lieux où s'exerce la Justice. Autant ces espaces sont familiers au juge de par sa formation, ses attributions et sa pratique, autant ils sont des territoires vierges pour le jeune qui y pénètre souvent pour la première fois. S’il peut être impressionné par l'architecture solennelle et les recoins réservés à la détention provisoire, il porte aussi son attention avec humour sur des détails incongrus passant inaperçus aux yeux des habitués :

Racontez l'évènement qui vous a le plus impressionné dans le passé)

13 mai 1953,

Ce qui m'a le plus impressionné est ceci : c'est lorsque pour la première fois je suis allé au palais de justice. Je ne l'imagine pas si grand qu'il est. Je le croyais moyen. Mais aussi de voir dans ce palais de justice une cathédrale que tous les étrangers viennent visiter et aussi qu'il y a au sous-sol un bar. Ily a dans ce palais de justice des grandes salles, où sur les portes il y a marqué "Petit parquet" on "Grand parquet". J'ai vu ça lorsque je suis allé voir monsieur le juge Pužin qui m'a lui envoyé 
dans un centre. Je ne pouvais croire que des garcons de mon âge allaient très souvent voir le juge devant pleins de gens, avec beaucoup de gardes, pour ne pas que je me sauve, mais je me suis fortement trompé. [...] Ce qui m'a beaucoup impressionné au palais de Justice, c'est de voir le dépôt où l'on voit de grosses portes où il y a des barreaux, et aussi j'ai vu des hommes accompagnés de gendarmes avec des menottes. Tout ceci c'était la première fois que je le voyais. »

Un des exercices d'écriture donné aux mineurs de Savigny propose un drôle de jeu de rôle, propre aux tests projectifs employés à l'époque, le jeune étant invité à assumer la position du juge. Outre le fait que cette rédaction est un excellent révélateur des motivations qui ont pu conduire à l'acte, on ne peut qu'être surpris parfois de leur bon sens, au point de se surprendre à rêver, ne serait-ce qu'une fois, que l'illusion fasse réalité :

octobre-décembre 1959

Si tu étais juge à quelle peine condamnerais-tu?

Un garçon qui est parti de chez lui ?

Une fugue souvent tient sa peine elle-même, car un adolescent qui s'enfuit, pour qu'il vive, il est souvent contraint à travailler, car vivre seul au dépend des autres n'est pas toujours facile, j'en déduis qu'il retournera au foyer, heureux de retrouver ses parents et ses choses familières. Beaucoup font des fugues amoureuses, mais la vie est vite impossible et il retourne aussi vite chez. lui et se contente des rendez-vous. Sauf exceptions.

Qui a volé $10.000 \mathrm{~F}$ pour la première fois?

Tout cela dépend des circonstances atténuantes, s'il a tout ce qu'il faut chez. lui, le plaisir de voler et de sentir le danger, est excusable, mais mérite une leçon pour apprendre à ne pas jouer avec le feu... Qui a volé une voiture?

Pour un jeune la chose est très excusable, car tout le monde comprend, du moins je le suppose, que sentir une mécanique vibrer sous ses doigts comme si elle dépendait uniquement de votre cerveau est quelque chose de merveilleux. Mais 5 jours de "tôle p pour calmer son ardeur à l'exaltation de la griserie de la vitesse, vivre au ralenti lui fera le plus grand bien.

Qui a blessé un vieillard pour le voler?

Cela est très complexe. S'il était proche de la folie, j'entends un être qui n'a rien mangé depuis une semaine et quelques jours, il se peut qu'il tombe lors d'une grisée accidentelle sur un vieillard. De là son état étant dans la demi inconscience reste à prowver. Sinon, sinon, en plus de la peur normale, je lui impliquerais un petit châtiment à ma façon: lunettes noires, coton dans les oreilles, mains liées et genouillères rigides, lui faisant voir les souffrances des personnes âgées. 